\title{
Análisis de coeficientes de sendero para el rendimiento de semillas en Bromus catharticus
}

\author{
Liliana A. Abbott ${ }^{1}$, Susana M. Pistorale y Olga S. Filippini \\ Departamento de Ciencias Básicas, Universidad Nacional de Luján. \\ CC 221, (6700) Luján, Buenos Aires, Argentina
}

\begin{abstract}
L. A. Abbott, S. M. Pistorale, and O. S. Filippini. 2007. Path coefficient analysis for the seed yield in Bromus catharticus. Cien. Inv. Agr. 34(2):141-149. Over a two-year period, 13 genotypes of rescue grass were evaluated in a completely randomized design with six replications. The correlation coefficients and the path coefficient were analysed over nine characters related to seed production. Genotypic variability was significant for total seeds per panicle in the second year $(p=0.019)$, flag leaf length was significant in both years $(p=0.039$ and 0.045 , respectively), and the number of tillers per plant was significant in the first year $(p=0.003)$. For the rest of the characters, variability was highly significant $(p \leq 0.001)$. The relationship between genotype and the environment interaction was significant for the following characteristics: the number of tillers per plant $(p=0.003)$, the number of panicles per plant $(p=0.010)$, the width of the flag leaf $(p=0.010)$ and the 1000 seeds weight $(p=0.047)$. For the remaining characters, the interaction was not significant. Path coefficient analyses demonstrated that the total number of seeds per panicle and the number of spikelets per panicle were the main components that determined seed yield. The contribution of these components was influenced by other characters, such as panicle and flag leaf lengths. Therefore, these can be considered to be secondary yield components. The following four components: the number of total seeds per panicle, the number of spikelets per panicle, the panicle length and the flag leaf length rendered a total determination for this system that explained $83.36 \%$ and $81.61 \%$ of the phenotypic variation for the number of fully developed seeds per panicle each year.
\end{abstract}

Key words: Phenotypic correlation, rescue grass, yields components.

\section{Introducción}

La cebadilla criolla (Bromus catharticus Vahl.) es uno de los pastos nativos más cultivado en Argentina y es una de las pocas especies forrajeras autóctonas que se cultiva en Bolivia, Brasil, Chile, Ecuador, Perú y Uruguay e inclusive en el Sur de los Estados Unidos.

Se caracteriza por poseer numerosas ramificaciones basales (macollos) algunas de las cuales producen cañas floríferas en cuyo extremo está la inflorescencia. Las

Recibido 26 diciembre 2006. Aceptado 15 mayo 2007.

'Dirigir correspondencia a L. Abbott: genetica@mail.unlu.edu.ar inflorescencias corresponden a panojas laxas con espiguillas achatadas, cada una de las cuales contiene de seis a doce semillas grandes. Posee dos tipos de floración, cleistógama y chasmógama. No obstante, se considera una especie autógama facultativa debido a su baja tasa de alogamia (Cladera and Pahlen 1984; Naranjo 1985; Pahlen 1986; Morant 1990; Aulicino y Arturi, 2002).

Es una especie anual o bianual de ciclo otoñoinvierno-primaveral con alta capacidad de implantación y buen crecimiento inicial. Se consocia muy bien con otras especies, formando pasturas de óptima calidad en las cuales perdura por muchos años a causa de su gran producción de semillas y su destacado comportamiento en la resiembra natural (Wolff et al., 1996). 
En cereales y oleaginosas, el mejoramiento genético convencional ha tenido un gran impacto en el incremento del rendimiento, calidad y resistencia a plagas y enfermedades (Evans, 1998). Sin embargo, los progresos en el mejoramiento de especies forrajeras han sido significativamente menores, especialmente en rendimiento (Snaydon, 1985; Brummer, 1999). Entre otros factores, esto obedece a la domesticación reciente, la complejidad de objetivos (por ejemplo, la mejora en producción de forraje y de semilla se contraponen por su correlación negativa), la inestabilidad del mercado y las menores inversiones realizadas en esta área. Sin embargo, en los últimos años, han aumentado los estudios para la producción de semilla mejorada en forrajeras, lo que es esencial para competir en el mercado de cultivares de Argentina.

La producción de semilla depende directa o indirectamente del número de caracteres agronómicos tales como altura de planta, área foliar, producción de materia seca, y ancho y largo de la hoja bandera (Grifiths 1965). Por lo tanto, es importante en la mejora de la producción de semillas forrajeras, tener una clara comprensión de las relaciones entre la producción de semilla y estos caracteres agronómicos.

A pesar de la utilidad de los coeficientes de correlación en la cuantificación de la magnitud y dirección de los efectos de factores en la determinación de caracteres complejos, estos coeficientes no indican la exacta importancia que tienen los efectos directos e indirectos que esos caracteres tienen sobre el rendimiento. Un coeficiente de correlación alto o bajo entre dos variables se puede deber al efecto de una tercera variable o grupo de variables (Singh y Chaudhary, 1977; Cruz y Regazzi, 1997; Vencovsky y Barriga, 1992). En estudios previos realizados con cebadilla criolla se determinó que no siempre el carácter mejor correlacionado con el rendimiento fue el que más contribuyó a su expresión final (Pistorale y Wolff, 1998).

El método analítico de coeficientes de sendero (path coefficientanalysis) permite descomponer las correlaciones entre dos variables (X e Y) en una suma del efecto directo de $\mathrm{X}$ sobre $\mathrm{Y}$ $\mathrm{y}$ los efectos indirectos de $\mathrm{X}$ sobre $\mathrm{Y}$ vía otras variables independientes en un sistema de correlaciones. El análisis de sendero tiene por objetivo identificar las posibles explicaciones causales de las correlaciones observadas entre una variable respuesta (dependiente) y una serie de variables predictoras (independientes) (Wright, 1921).

En la mejora de plantas, se han desarrollado varios trabajos con el apoyo de este análisis, siendo de gran importancia para el mejorador en la formulación de procedimientos apropiados para la selección (Dewey y Lu, 1959; Milligan et al., 1990). Por lo tanto, el objetivo de este trabajo fue analizar los componentes del rendimiento de semillas en $B$. catharticus mediante el análisis de coeficientes de sendero.

\section{Materiales y métodos}

\section{Materiales}

El comportamiento reproductivo de poblaciones naturales de cebadilla criolla se iniciaron estudios en 1990 en las localidades de Luján, Gowland y Alberti $\left(59^{\circ} 60^{\prime}, 59^{\circ} 23^{\prime}\right.$ y $60^{\circ} 16^{\prime}$ de longitud y $34^{\circ} 34^{\prime}, 34^{\circ} 41^{\prime}$ y $35^{\circ} 10^{\prime}$ de latitud sur, respectivamente) de la provincia de Buenos Aires.

De cada población se seleccionaron in situ las plantas que mostraron el mejor comportamiento en los siguientes caracteres: 1. Número de macollos por planta, 2. Número de panojas por planta, 3. Capacidad de supervivencia y 4 . Calidad de la semilla, estimada por el peso y poder germinativo. Para estimar parámetros genéticos, la descendencia de estas plantas se sometieron a pruebas de progenie entre $1994 \mathrm{y}$ 1998 (Wolff et al., 2006).

Los caracteres estudiados fueron: 1. Número de macollos por planta, determinado antes que se transformaran en macollos reproductivos. 2 . Número de panojas por planta determinados a medida que se cosecharon. 3. Largo de panoja, medido desde la inserción hasta el extremo. 4. Número de espiguillas por panoja. 5. Longitud de la hoja bandera, medida desde su inserción hasta el extremo. 6. Ancho de hoja bandera, 
medida en su parte media. 7. Número de semillas totales y semillas llenas por panoja, para lo cual se trillaron manualmente tres panojas por planta. Se contaron todas sus semillas lo que se utilizó para estimar el rendimiento por planta. 9. Peso de 1000 semillas, estimado en una muestra de 200 semillas llenas. Las panojas se cosecharon a medida que maduraron, y se guardaron separadamente en bolsas de papel hasta evaluarlas en el laboratorio. Se calculó el valor promedio del largo de panoja, largo y ancho de hoja bandera y número espiguillas de tres panojas por planta.

\section{Diseño y análisis estadístico}

Para este trabajo se seleccionaron 13 genotipos, los que se evaluaron durante 2001 y 2002. El trabajo se diseñó en un experimento completamente aleatorizado con seis repeticiones por genotipo. Las unidades experimentales consistieron en macetas con plantas individuales ubicadas en el campo experimental de la Universidad de Luján, Buenos Aires, Argentina.

Las correlaciones fenotípicas (r) se calcularon utilizando el coeficiente de correlación de Pearson, como medida de la magnitud de la asociación lineal entre dos variables que no depende de las unidades de medida de las variables originales. El coeficiente de sendero (path analysis) se determinó con el software InfoStat (Estadística y Biometría, Facultad de Ciencias Agropecuarias, Universidad Nacional de Córdoba, Argentina). El coeficiente de determinación $\left(\mathrm{R}^{2}\right)$ del carácter rendimiento se calculó como la sumatoria del producto del efecto directo por el coeficiente de correlación de la variable dependiente y la variable componente. Si el coeficiente de determinación no explicó el $100 \%$ de la variación en el rendimiento, se incluyó un componente para considerar efectos residuales (E) no previstos con los caracteres analizados.

\section{Resultados y discusión}

\section{Resultados estadísticos descriptivos}

En el Cuadro 1 se muestran las medias, los valores máximos, mínimos y los coeficientes de variación para todos los caracteres estudiados. El número de semillas llenas por panoja y el

Cuadro 1. Media, valores mínimos, máximos y coeficientes de variación para diferentes caracteres en cebadilla criolla (Bromus catharticus) en dos años de estudio.

Table 1. Mean, minimum, maximum values and coefficient of variation for different characters in rescue grass (Bromus catharticus) obtained in two years of study.

\begin{tabular}{|c|c|c|c|c|c|}
\hline Carácter & Año & Media & Mínimo & Máximo & $\mathrm{CV}^{1}, \%$ \\
\hline \multirow[t]{2}{*}{$\mathrm{N}^{o}$ de semillas llenas/panoja } & 2001 & 162,00 & 57,67 & 280,67 & 31,52 \\
\hline & 2002 & 144,31 & 38,33 & 290,33 & 38,51 \\
\hline \multirow[t]{2}{*}{$\mathrm{N}^{\mathrm{o}}$ de semillas totales/panoja } & 2001 & 198,72 & 78,00 & 379,50 & 30,52 \\
\hline & 2002 & 169,38 & 40,67 & 325,00 & 38,78 \\
\hline \multirow[t]{2}{*}{$\mathrm{N}^{\mathrm{o}}$ de espiguillas/panoja } & 2001 & 40,53 & 27,00 & 71,00 & 20,31 \\
\hline & 2002 & 40,14 & 17,00 & 89,00 & 28,70 \\
\hline \multirow[t]{2}{*}{ Largo de panoja, $\mathrm{cm}$} & 2001 & 28,68 & 23,33 & 38,17 & 9,97 \\
\hline & 2002 & 24,41 & 15,67 & 31,83 & 14,13 \\
\hline \multirow[t]{2}{*}{ Largo de hoja bandera, $\mathrm{cm}$} & 2001 & 23,45 & 17,67 & 31,17 & 10,66 \\
\hline & 2002 & 9,88 & 13,00 & 31,50 & 17,96 \\
\hline \multirow[t]{2}{*}{ Peso de 1000 semillas, $\mathrm{cm}$} & 2001 & 9,94 & 6,65 & 13,60 & 16,80 \\
\hline & 2002 & 10,43 & 6,14 & 15,60 & 16,87 \\
\hline \multirow[t]{2}{*}{ Ancho de hoja bandera, $\mathrm{cm}$} & 2001 & 0,67 & 0,43 & 0,93 & 16,42 \\
\hline & 2002 & 0,68 & 0,43 & 1,03 & 19,12 \\
\hline \multirow[t]{2}{*}{$\mathrm{N}^{o}$ de panojas/planta } & 2001 & 9,59 & 3,00 & 15,00 & 25,65 \\
\hline & 2002 & 11,71 & 3,00 & 25,00 & 46,03 \\
\hline \multirow[t]{2}{*}{$\mathrm{N}^{\mathrm{o}}$ de macollos/planta } & 2001 & 18,09 & 5,00 & 30,00 & 27,69 \\
\hline & 2002 & 20,49 & 9,00 & 36,00 & 29,97 \\
\hline
\end{tabular}

${ }^{1} \mathrm{CV}$, coeficiente de variación.

${ }^{I} \mathrm{CV}$, coefficient of variation. 
número de semillas totales por panoja fueron mayores en el primer año con respecto al segundo año de este estudio. Estas diferencias se podrían deber a las mayores lluvias ocurridas en octubre de 2001 respecto a 2002, época en que queda determinado el número de semillas. Para el resto de los caracteres, los valores medios fueron similares en los dos años.

En los dos años de estudio, los coeficientes de variación $(\mathrm{CV})$ oscilaron entre 10 y $46 \%$ para el largo y número de panoja, respectivamente. Comparando ambos años, la menor diferencia entre los CV fue para peso de 1000 semillas y las mayores fueron para número de panojas por planta y largo de la hoja bandera. De acuerdo con los coeficientes de variación obtenidos existió variabilidad, lo que permite suponer que es posible obtener buenos resultados en el mejoramiento de esta especie por selección. No obstante, la mayor proporción de la variancia genética de esta especie estuvo asociada a la interacción entre el genotipo y el ambiente (Wolff et al., 1996).

\section{Análisis de la variancia}

Mediante un análisis de varianza se calculó la variabilidad de los genotipos para los distintos caracteres (Cuadro 2). Esta variabilidad fue significativa para número de semillas totales por panoja en el 2002, largo de hoja bandera en los dos años de estudio y número de macollos por planta en el 2001. Para el resto de los caracteres evaluados la variabilidad fue significativa a $\mathrm{p} \leq 0,001$.

Además, el análisis de varianza permitió estimar la interacción entre el genotipo y el ambiente (Cuadro 2). Esta interacción genético x ambiental se definió como la respuesta diferencial para un grupo de genotipos frente a distintos ambientes. Su análisis resultó de interés para el desarrollo de las distintas etapas de evaluación y selección de materiales. En este estudio, los años fueron considerados como ambientes diferentes debido a las variaciones climáticas, especialmente en precipitaciones y temperaturas. La interacción genotipo $\mathrm{x}$ ambiente fue significativa únicamente para número de macollos por planta $(\mathrm{p}=0,003)$, número de panojas por planta $(\mathrm{p}=0,01)$, ancho de hoja bandera $(\mathrm{p}=0,01)$
Cuadro 2. Variabilidad genotípica e interacción genotipo $\mathrm{x}$ ambiente para diferentes caracteres en cebadilla criolla (Bromus catharticus) en dos años de estudio.

Table 2. Genotypic variability and genotype $(G) x$ environment $(E)$ interaction for different characters in rescue grass (Bromus catharticus) in two years of study.

\begin{tabular}{|c|c|c|c|}
\hline \multirow[t]{2}{*}{ Carácter } & \multicolumn{2}{|c|}{ Variabilidad genotípica } & \multirow{2}{*}{$\begin{array}{c}\text { Interacción } \\
\text { Gx E } \\
\text { Cuadrado } \\
\text { medio }\end{array}$} \\
\hline & $\begin{array}{c}\text { Cuadrado } \\
\text { medio } \\
2001\end{array}$ & $\begin{array}{c}\text { Cuadrado } \\
\text { medio } \\
2002\end{array}$ & \\
\hline $\mathrm{N}^{\mathrm{o}}$ de semillas & 9514,24 & 8515,72 & 2138,15 \\
\hline 1lenas/panoja & $(<0,000)^{1}$ & $(<0,000)^{1}$ & $(0,256)^{1}$ \\
\hline $\mathrm{N}^{\mathrm{o}}$ de semillas & 9100,08 & 8109,79 & 2775,56 \\
\hline totales/panoja & $(0,001)$ & $(0,019)$ & $(0,567)$ \\
\hline $\mathrm{N}^{\mathrm{o}} \mathrm{de}$ & 254,29 & 418,23 & 95,31 \\
\hline espiguillas/panoja & $(<0,000)$ & $(<0,000)$ & $(0,077)$ \\
\hline Largo de panoja & $\begin{array}{c}23,66 \\
(<0,000)\end{array}$ & $\begin{array}{c}30,58 \\
(<0,000)\end{array}$ & $\begin{array}{c}6,08 \\
(0,570)\end{array}$ \\
\hline $\begin{array}{l}\text { Largo de hoja } \\
\text { bandera }\end{array}$ & $\begin{array}{c}10,81 \\
(0,039)\end{array}$ & $\begin{array}{c}21,65 \\
(0,045)\end{array}$ & $\begin{array}{c}11,92 \\
(0,156)\end{array}$ \\
\hline $\begin{array}{l}\text { Peso de } 1000 \\
\text { semillas }\end{array}$ & $\begin{array}{c}9,47 \\
(<0,000)\end{array}$ & $\begin{array}{c}14,01 \\
(<0,000)\end{array}$ & $\begin{array}{c}2,44 \\
(0,047)\end{array}$ \\
\hline $\begin{array}{l}\text { Ancho de hoja } \\
\text { bandera }\end{array}$ & $\begin{array}{c}0,03 \\
(<0,000)\end{array}$ & $\begin{array}{c}0,06 \\
(<0,000)\end{array}$ & $\begin{array}{c}0,02 \\
(0,010)\end{array}$ \\
\hline $\begin{array}{l}\mathrm{N}^{\circ} \text { de panojas/ } \\
\text { planta }\end{array}$ & $\begin{array}{c}21,52 \\
(<0,000)\end{array}$ & $\begin{array}{c}86,44 \\
(<0,000)\end{array}$ & $\begin{array}{c}25,21 \\
(0,010)\end{array}$ \\
\hline $\begin{array}{l}\mathrm{N}^{\circ} \text { de macollos/ } \\
\text { planta }\end{array}$ & $\begin{array}{c}56,43 \\
(0,003)\end{array}$ & $\begin{array}{c}109,01 \\
(<0,000)\end{array}$ & $\begin{array}{c}58,10 \\
(0,003)\end{array}$ \\
\hline
\end{tabular}

${ }^{1}$ Valores probables entre paréntesis.

'Probability values are indicated between brackets.

y peso de 1000 semillas $(p=0,047)$. Estos resultados demostraron que los caracteres más plásticos (aquellos que mostraron una respuesta diferencial en distintos ambientes) fueron componentes del rendimiento en semillas. No obstante, el rendimiento, probablemente por la compensación de sus caracteres componentes, no mostró una interacción genotipo $\mathrm{x}$ ambiente estadísticamente significativa (Pistorale et al. 2004).

\section{Correlaciones fenotípicas}

En los dos años, la correlación entre el rendimiento en número de semillas llenas por panoja y el número de semillas totales por panoja fue positiva y alta $(0,889$ y 0,900$)$ (Cuadro 3). La correlación entre el número de semillas llenas por panoja y el número de espiguillas por panoja también fue positiva y alta en el $2001(0,672)$ y moderada en el 2002 $(0,358)$. Con el largo de panoja, la correlación fue moderada en el $2001(0,250)$ y alta en el 
$2002(0,577)$ y, con el largo de hoja bandera, fue moderada en ambos años $(0,249$ y 0,386$)$. Estas correlaciones fueron significativas a $\mathrm{p}<0,05$.

Con respecto a las correlaciones entre los diferentes componentes, el número de semillas totales por panoja tuvo una correlación alta y positiva con el número de espiguillas por panoja en el primer año $(0,565)$ y moderada en el segundo $(0,297)$. Con el largo de panoja fue moderada en el primer año $(0,229)$ y alta en el segundo $(0,574)$, mientras que con el largo de hoja bandera, la correlación fue moderada en los dos años $(0,242$ y 0,384$)$. También estas correlaciones fueron significativas a $\mathrm{p}<0,05$.
En el 2001, el peso de 1000 semillas presentó correlaciones negativas con el número de semillas llenas por panoja $(-0,408)$, el número de semillas totales por panoja $(-0,467)$ y el número de espiguillas por panoja $(-0,205)$ (Cuadro 3$)$. En el 2002, estas correlaciones fueron positivas para el número de semillas llenas por panoja $(0,245)$, semillas totales por panoja $(0,301)$ pero fue negativa para el número de espiguillas por panoja $(-0,123)$ (Cuadro 3). Estas variaciones en el signo de las correlaciones probablemente se debieron a las mayores lluvias caídas en diciembre de 2002. Para el resto de los caracteres las correlaciones fueron variadas en signo y magnitud.

Cuadro 3. Correlaciones fenotípicas entre los caracteres en cebadilla criolla (Bromus catharticus) en dos años de estudio. Table 3. Phenotypic correlation between the characters in rescue grass (Bromus catharticus) in two years of study.

\begin{tabular}{|c|c|c|}
\hline Caracteres & $2001(\mathrm{p})$ & $2002(\mathrm{p})$ \\
\hline $\mathrm{N}^{\circ}$ de semillas llenas/panoja $-\mathrm{N}^{\circ}$ de semillas totales/panoja & $0,889(<0,0001)$ & $0,900(0,000)$ \\
\hline $\mathrm{N}^{o}$ de semillas llenas/panoja - $\mathrm{N}^{\circ}$ de espiguillas/panoja & $0,672(0,000)$ & $0,358(0,000)$ \\
\hline $\mathrm{N}^{o}$ de semillas llenas/panoja - largo de panoja & $0,250(0,028)$ & $0,577(0,000)$ \\
\hline $\mathrm{N}^{o}$ de semillas llenas/panoja - largo de hoja bandera & $0,249(0,029)$ & $0,386(0,001)$ \\
\hline $\mathrm{N}^{\mathrm{o}}$ de semillas llenas/panoja - peso de 1000 semillas & $-0,408(<0,0001)$ & $0,245(0,032)$ \\
\hline $\mathrm{N}^{\mathrm{o}}$ de semillas llenas/panoja - ancho de hoja bandera & $-0,098(0,399)$ & $0,191(0,096)$ \\
\hline $\mathrm{N}^{o}$ de semillas llenas/panoja - $\mathrm{N}^{\circ}$ de panoja/planta & $0,043(0,710)$ & $0,160(0,165)$ \\
\hline $\mathrm{N}^{o}$ de semillas llenas/panoja - $\mathrm{N}^{\mathrm{o}}$ de macollos/planta & $-0,124(0,284)$ & $0,015(0,896)$ \\
\hline $\mathrm{N}^{\mathrm{o}}$ de semillas totales/panoja $-\mathrm{N}^{\circ}$ de espiguillas/panoja & $0,565(0,000)$ & $0,297(0,009)$ \\
\hline $\mathrm{N}^{o}$ de semillas totales/panoja - largo de panoja & $0,229(0,045)$ & $0,574(0,000)$ \\
\hline $\mathrm{N}^{o}$ de semillas totales/panoja - largo de hoja bandera & $0,242(0,034)$ & $0,384(0,001)$ \\
\hline $\mathrm{N}^{\mathrm{o}}$ de semillas totales/panoja - peso de 1000 semillas & $-0,467(<0,0001)$ & $0,311(0,006)$ \\
\hline $\mathrm{N}^{\mathrm{o}}$ de semillas totales/panoja - ancho de hoja bandera & $-0,039(0,735)$ & $0,227(0,047)$ \\
\hline $\mathrm{N}^{o}$ de semillas totales/panoja - $\mathrm{N}^{\mathrm{o}}$ de panoja/planta & $-0,074(0,522)$ & $0,057(0,621)$ \\
\hline $\mathrm{N}^{o}$ de semillas totales/panoja $-\mathrm{N}^{\circ}$ de macollos/planta & $-0,194(0,091)$ & $0,100(0,388)$ \\
\hline $\mathrm{N}^{o}$ de espiguillas/panoja - largo de panoja & $0,195(0,089)$ & $0,333(0,003)$ \\
\hline $\mathrm{N}^{o}$ de espiguillas/panoja - largo de hoja bandera & $0,238(0,037)$ & $0,295(0,009)$ \\
\hline $\mathrm{N}^{\mathrm{o}}$ de espiguillas/panoja - peso de 1000 semillas & $-0,205(0,073)$ & $-0,123(0,285)$ \\
\hline $\mathrm{N}^{o}$ de espiguillas/panoja - ancho de hoja bandera & $-0,122(0,289)$ & $0,137(0,235)$ \\
\hline $\mathrm{N}^{o}$ de espiguillas/panoja - $\mathrm{N}^{\mathrm{o}}$ de panoja/planta & $0,060(0,601)$ & $0,349(0,0029$ \\
\hline $\mathrm{N}^{o}$ de espiguillas/panoja - $\mathrm{N}^{\mathrm{o}}$ de macollos/planta & $-0,141(0,221)$ & $0,259(0,023)$ \\
\hline Largo de panoja - largo de hoja bandera & $0,616(0,000)$ & $0,703(0,000)$ \\
\hline Largo de panoja - peso de 1000 semillas & $0,159(0,166)$ & $0,488(0,000)$ \\
\hline Largo de panoja - ancho de hoja bandera & $0,452(0,000)$ & $0,524(0,000)$ \\
\hline Largo de panoja - $\mathrm{N}^{\circ}$ de panojas/planta & $-0,175(0,127)$ & $0,043(0,710)$ \\
\hline Largo de panoja - $\mathrm{N}^{\circ}$ de macollos/planta & $-0,178(0,120)$ & $0,147(0,201)$ \\
\hline Largo de hoja bandera - peso de 1000 semillas & $0,117(0,312)$ & $0,254(0,026)$ \\
\hline Largo de hoja bandera - ancho de hoja bandera & $0,522(0,000)$ & $0,625(0,000)$ \\
\hline Largo de hoja bandera - $\mathrm{N}^{\circ}$ de panojas/planta & $-0,226(0,048)$ & $0,104(0,369)$ \\
\hline Largo de hoja bandera - $\mathrm{N}^{\circ}$ de macollos/planta & $-0,188(0,102)$ & $0,157(0,171)$ \\
\hline Peso de 1000 semillas - ancho de hoja bandera & $0,341(0,002)$ & $0,481(0,000)$ \\
\hline Peso de 1000 semillas - $\mathrm{N}^{\circ}$ de panojas/planta & $0,011(0,923)$ & $0,027(0,815)$ \\
\hline Peso de 1000 semillas - $\mathrm{N}^{\circ}$ de macollos/planta & $-0,146(0,207)$ & $0,087(0,454)$ \\
\hline Ancho de hoja bandera - $\mathrm{N}^{\circ}$ de panojas/planta & $-0,179(0,120)$ & $0,226(0,048)$ \\
\hline Ancho de hoja bandera - $\mathrm{N}^{\circ}$ de macollos/planta & $-0,304(0,007)$ & $0,444(0,000)$ \\
\hline $\mathrm{N}^{\mathrm{o}}$ de macollos/planta - $\mathrm{N}^{\mathrm{o}}$ de panojas/planta & $0,278(0,014)$ & $0,409(0,000)$ \\
\hline
\end{tabular}


Cuadro 4. Coeficientes de sendero que muestran los efectos directos e indirectos de los componentes del rendimiento en cebadilla criolla (Bromus catharticus).

Table 4. Path coefficients showing direct and indirect effects of the components yield in rescue grass (Bromus catharticus).

\begin{tabular}{|c|c|c|}
\hline \multirow[t]{2}{*}{ Vías de asociación } & \multicolumn{2}{|c|}{ Valores estimados en } \\
\hline & 2001 & 2002 \\
\hline \multicolumn{3}{|l|}{ Rendimiento en semillas llenas vs. Espiguillas } \\
\hline Efecto directo & 0,25 & 0,09 \\
\hline Efecto indirecto vía semillas totales & 0,42 & 0,25 \\
\hline Efecto indirecto vía largo de panoja & 0,01 & 0,03 \\
\hline Efecto indirecto vía largo de hoja bandera & 0,01 & 0,01 \\
\hline $\mathrm{r}$ total & 0,67 & 0,36 \\
\hline \multicolumn{3}{|c|}{ Rendimiento en semillas llenas vs. semillas totales } \\
\hline Efecto directo & 0,74 & 0,83 \\
\hline Efecto indirecto vía espiguillas & 0,14 & 0,03 \\
\hline Efecto indirecto vía largo de panoja & 0,01 & 0,04 \\
\hline Efecto indirecto vía largo de hoja bandera & 0,01 & 0,01 \\
\hline $\mathrm{r}$ total & 0,89 & 0,90 \\
\hline \multicolumn{3}{|c|}{ Rendimiento en semillas llenas vs. largo de panoja } \\
\hline Efecto directo & 0,04 & 0,07 \\
\hline Efecto indirecto vía espiguillas & 0,05 & 0,07 \\
\hline Efecto indirecto vía semillas totales & 0,17 & 0,48 \\
\hline Efecto indirecto vía largo de hoja bandera & 0,01 & $-0,01$ \\
\hline $\mathrm{r}$ total & 0,25 & 0,58 \\
\hline \multicolumn{3}{|c|}{ Rendimiento en semillas llenas vs. largo de hoja bandera } \\
\hline Efecto directo & $-0,01$ & $-0,01$ \\
\hline Efecto indirecto vía espiguillas & 0,06 & 0,03 \\
\hline Efecto indirecto vía semillas totales & 0,18 & 0,32 \\
\hline Efecto indirecto vía largo de panoja & 0,03 & 0,05 \\
\hline $\mathrm{r}$ total & 0,25 & 0,39 \\
\hline Determinación total del carácter, $\mathrm{R}^{2}(\%)$ & 83,36 & 81,61 \\
\hline Efectos residuales, E (\%) & 16,64 & 18,39 \\
\hline
\end{tabular}

A veces las correlaciones simples entre los componentes del rendimiento resultan poco informativas respecto de la relación funcional entre componentes de distinta jerarquía. La metodología del análisis de coeficientes de sendero, utilizada en este trabajo, proporcionó una aproximación a lo que pueden ser las estrategias biológicas de la determinación de rendimiento en semillas u otros caracteres complejos.

\section{Análisis del coeficiente de sendero}

El sistema con cuatro componentes: número de semillas totales por panoja, número de espiguillas por panoja, largo de panoja y largo de hoja bandera dio una determinación total del sistema $\left(\mathrm{R}^{2}\right)$ que explicó el 83,36 y 81,61\% de la variación fenotípica para el número de semillas llenas por panoja en 2001 y 2002, respectivamente (Cuadro 4). La inclusión de más caracteres en el modelo dio $\mathrm{R}^{2}$ levemente más altos. Sin embargo, aun con el total de los caracteres estudiados el $\mathrm{R}^{2}$ explicó el 84,28 y $85,09 \%$ de la variación fenotípica en 2001 y 2002, respectivamente.

Por lo tanto, el sistema con cuatro caracteres componentes se eligió para mantener el modelo tan simple como fuera posible. Es importante considerar que ésta es una aproximación simplificada de un conjunto de procesos complejos de las plantas. Por este motivo, éste puede modificarse al incluir nuevos componentes en el sistema. Según Allard y Bradshaw (1964) los avances en el mejoramiento se producen al considerar caracteres finales, tales como rendimiento o calidad. Por lo tanto, el progreso real en el mejoramiento genético de 
una especie solo sería posible si se definieran los componentes que conducen a ese carácter final. Aún reconociendo sus limitaciones, resulta de interés este tipo de análisis, el que atendiendo al conocimiento de las relaciones funcionales entre los componentes del rendimiento, puede mejorar la eficiencia de la selección en especies forrajeras (Pistorale et al., 1998).

\section{Efectos directos e indirectos}

El efecto directo del número de semillas totales por panoja sobre el rendimiento, fue muy alto en 2001 y 2002 (0,74 y 0,83, respectivamente). El del número de espiguillas por panoja fue moderado en el 2001 pero bajo en el $2002(0,25$ y 0,09 , respectivamente). Los demás caracteres tuvieron un efecto directo muy bajo y en algunos casos negativos. Según Hill y Watkin (1975) el rendimiento final en varias especies forrajeras, incluida $B$. catharticus, parece depender más de la polinización, fertilización y formación de semillas que de la relación entre macollos y panojas. Estas observaciones coinciden con las realizadas por Wolff et al. (1996).

El efecto indirecto del número espiguillas por panoja con el número de semillas totales por panoja fue alto en $2001(0,42)$ y moderado en $2002(0,25)$. El efecto indirecto del largo de panoja sobre semillas totales fue alto en 2002 $(0,48)$ y moderado en $2001(0,17)$. El largo de hoja bandera tuvo un efecto indirecto alto $(0,32)$ sobre el número de semillas totales en el 2002 pero moderado en $2001(0,18)$. También fue moderado el efecto indirecto del número de semillas totales por panoja sobre el número de espiguillas por panoja $(0,14)$ en 2001 . El resto de los efectos indirectos fueron muy bajos y en algunos casos negativos.

Según los resultados obtenidos, se puede apreciar que los cuatro caracteres estudiados como componentes del rendimiento incidieron de forma diferente y directa sobre el rendimiento. El número de semillas totales de espiguillas por panoja tuvieron un alto efecto directo, positivo sobre el rendimiento. Los efectos directos del largo de panoja y del largo de hoja bandera fueron bajos, positivo y negativo, respectivamente. Esto permitió considerar a los primeros como componentes principales y a los segundos, como componentes secundarios del rendimiento (Fonseca y Patterson, 1968). Los componentes secundarios influyeron sobre los componentes principales y, a través de ellos, sobre el rendimiento, estableciendo un sistema de causa y efecto (Parodi et al., 1970).

Los resultados obtenidos en este trabajo demostraron la inconveniencia de usar solo correlaciones simples para estudiar el grado de asociación e interacción entre los caracteres componentes del rendimiento en semillas en cebadilla criolla. El análisis de los coeficientes de sendero tuvo mayor utilidad, permitiendo conocer la contribución real de un grupo de variables independientes sobre una variable dependiente por medio de sus efectos directos e indirectos. Al mismo tiempo el análisis de coeficientes de sendero mostró que el número de semillas totales y espiguillas por panoja fueron los componentes principales que determinaron el rendimiento en semilla en cebadilla criolla. La contribución de estos componentes dependió de otros caracteres como el largo de la panoja y de la hoja bandera. Estos últimos se pueden considerar componentes secundarios del rendimiento. La significativa interacción entre el genotipo y el ambiente obtenida para algunos caracteres, componentes del rendimiento, como asimismo las diferencias climáticas existentes en los años de este estudio, sugieren la necesidad de probar los genotipos en más de una temporada para la correcta planificación de un programa de mejoramiento en cebadilla criolla (B. catharticus).

\section{Resumen}

Trece genotipos de Bromus catharticus (cebadilla criolla) fueron evaluados para rendimiento en semilla en 2001 y 2002, usando un diseño completamente aleatorizado con seis repeticiones por genotipo. Se analizaron las correlaciones y el coeficiente de sendero para nueve caracteres relacionados con la producción de semilla. La variabilidad genotípica fue estadísticamente significativa para número de semillas totales por panoja en el segundo año $(\mathrm{p}=0,019)$, largo de hoja bandera en los dos años ( $p=0,039$ y 0,045$)$ y número de macollos por planta en el primer año $(p=0,003)$. La interacción genotipo $x$ ambiente 
fue significativa para número de macollos por planta $(\mathrm{p}=0,003)$, número de panojas por planta $(\mathrm{p}=0,010)$, ancho de hoja bandera $(\mathrm{p}=0,010)$ y peso de 1000 semillas $(\mathrm{p}=0,047)$. El análisis de coeficientes de sendero mostró que el número de semillas totales el número de espiguillas por panoja fueron los componentes principales que determinaron el rendimiento en semilla. La contribución de estos componentes dependió de otros caracteres, tales como largo de panoja y largo de hoja bandera. Estos últimos caracteres se pueden considerar componentes secundarios del rendimiento. El sistema con cuatro componentes representado por el número de semillas totales, número de espiguillas, y largo de panoja más el largo de hoja bandera explicó el 83,36 y 81,61\% de la variación fenotípica para el número de semillas llenas por panoja en los dos años de estudio.

Palabras claves: Cebadilla criolla, componentes del rendimiento, correlaciones fenotípicas.

\section{Agradecimientos}

Se agradece el financiamiento recibido del Departamento de Ciencias Básicas de la Universidad Nacional de Luján, Buenos Aires, Argentina para la realización de este trabajo.

\section{Literatura citada}

Allard, R.W., and A.D. Bradshaw. 1964. Implications of genotype-environment interactions in applied plant breeding. Crop Science 4:503-508

Aulicino, M., and M. Arturi. 2002. Phenotypic diversity in Argentinean populations of Bromus catharticus (Poaceae). Genetic and environmental components of quantitative traits. New Zealand Journal of Botany 40:223 -234 .

Bean, E.W. 1972. Clonal evaluation for increased seed production in two species of forage grasses, Festuca arundinacea Schreb. and Phleum pratense L. Euphytica 21:377-383.

Brummer, E.C. 1999. Capturing heterosis in forage crop cultivar development. Crop Science 39:943-954.

Cladera, J.L., and A. Von der Pahlen. 1984. Genetic and population study of esterases on Bromus catharticus Vahl. Boletín de Genética del Instituto Fitotécnico de Castelar (Argentina) 12:25-30.
Cruz, C.D. y A. Regazzi. 1997. Modelos Biométricos Aplicados ao Melhoramento Genético. Segunda ed. Universidad Federativa de Vi ऽosa. Imprenta Universitaria. Brasil. 390 pp.

Dewey, D.R., and K.H. Lu. 1959. A correlation and path-analysis of components of crested wheat grass seed production. Agronomy Journal 51:515-518.

Evans, L.T. 1998. Feeding Ten Billion: Plants and Population Growth. Cambridge. University Press Cambridge. UK. 264 pp.

Fonseca, S., and F.L. Patterson. 1968. Yield component heritabilities and interrelationships in winter wheat (Triticum aestivum L.). Crop. Science 8:614-617.

Griffiths, D.J. 1965. Breeding for higher seed yield from herbage varieties. Journal of the National Institute of Agricultural Botany 10:320-331.

Hamid, Z.A., and J.E. Grafius. 1978. Developmental allometry and its implication to grain yield in barley. Crop Science 18:83-86.

Hill, M.J., and B.R. Watkin. 1975. Seed production studies on perennial ryegrass, timothy and prairie grass. I. Effect of tiller age on tiller survival, ear emergence and seed head components. Journal of the British Grassland Society 30:63-71.

Mariotti, J.A. 1986. Fundamentos de Genética Biométrica. Aplicaciones al Mejoramiento Genético Vegetal. Colección de Monografías Científicas $\mathrm{N}^{\mathrm{o}}$ 32. OEA, Programa Regional de desarrollo Científico y Tecnológico. Washington, D.C. 149 pp.

Milligan, S.B., K.A. Gravois, K.P. Bischoff, and F.A. Martin, 1990. Crop effects on genetic relationships among sugarcane traits. Crop Science 30:927-931.

Morant, A. 1990. Determinación del porcentaje de fecundación cruzada en cebadilla criolla (Bromus catharticus Vahl.). Tesis de Magister, Universidad Nacional de Rosario, INTA Pergamino, Argentina. 99 pp.

Naranjo, C.A. 1985. Estudios citogenéticos, bioquímicos y sistemáticos en algunas especies americanas del género Bromus (Gramineae). Tesis de Doctorado, Universidad Nacional de Buenos Aires, Argentina. 243 pp.

Pahlen, A.W. Von der 1986. Evaluation of genetic variability of some native forage plants. Boletín de Genética del Instituto Fitotécnico de Castelar (Argentina) 4:1-6.

Parodi, P.C., F.L. Patterson, y W.E. Nyquist. 1970. Interrelaciones entre los componentes principales y secundarios de rendimiento en trigo, Triticum estivum L. Fitotecnia Latinoamericana 7:1-15.

Pistorale, S., and R. Wolff. 1998. Seed yield 
components in natural populations of Bromus catharticus Vahl (cebadilla criolla). J. Genetic and Breeding 52:223-231.

Pistorale, S., L. Abbott y R. Wolff. 2004. Variación en la expresión de caracteres reproductivos en cebadilla criolla durante dos años. $27^{\circ}$ Congreso Argentino de Producción Animal. Tandil, Argentina.

Snaydon, R. 1985. Aspects of the ecological genetics of pasture species. Pages 127-152. In: J. Haeck and J.W. Woldendorp (eds.) Structure and Functioning of Plant Populations. Editorail North Holland, Amsterdam. The Netherlands.

Singh, R.K., and B.D. Chaudhary. 1977. Biometrical Methods in Quantitative Genetics Analysis. Ludhiana: Kaliani Publishers. New Delhi. 288 pp.
Vencovsky, R., and P. Barriga. 1992. Genética Biométrica no Fitomelhoramento. Sociedad Brasileira de Genética. Ribeirao Preto, Brasil. $496 \mathrm{pp}$.

Wolff, R., L. Abbott, and S. Pistorale. 1996: Reproductive behaviour of Bromus catharticus Vahl. (Cebadilla criolla) in natural and cultivated populations. Journal of Genetics and Breeding 50:121-128.

Wolff, R., L. Abbott, and S. Pistorale. 2006: Estimation of genetic parameters in Bromus catharticus Vahl. Journal of Basic and Applied Genetics 17:51-59.

Wright, S. 1921. Correlation and causation. Journal of Agriculture Research 20:557-585. 